\title{
Time dependent behavior of a localized electron at a heterojunction boundary of graphene
}

\author{
Min Seok Jang, ${ }^{1}$ Hyungjun Kim, ${ }^{2,3}$ Harry A. Atwater, ${ }^{1, a)}$ and William A. Goddard III ${ }^{2,3, b)}$ \\ ${ }^{1}$ Thomas J. Watson Laboratories of Applied Physics, California Institute of Technology, \\ MC 128-95, Pasadena, California 91125, USA \\ ${ }^{2}$ Materials and Process Simulation Center, Beckman Institute, California Institute of Technology, \\ MC 139-74, Pasadena, California 91125, USA \\ ${ }^{3}$ Center for Materials Simulations and Design, Graduate School of EEWS, Korea Advanced Institute of \\ Science and Technology, Daejeon 305-701, Republic of Korea
}

(Received 11 February 2010; accepted 25 May 2010; published online 29 July 2010)

\begin{abstract}
We develop a finite-difference time-domain (FDTD) method for simulating the dynamics of graphene electrons, denoted GraFDTD. We then use GraFDTD to study the temporal behavior of a single localized electron wave packet, showing that it exhibits optical-like dynamics including the Goos-Hänchen effect [F. Goos and H. Hänchen, Ann. Phys. 436, 333 (1947)] at a heterojunction, but the behavior is quantitatively different than for electromagnetic waves. This suggests issues that must be addressed in designing graphene-based electronic devices analogous to optical devices. GraFDTD should be useful for studying such complex time-dependent behavior of a quasiparticle in graphene. () 2010 American Institute of Physics. [doi:10.1063/1.3454909]
\end{abstract}

The graphene two-dimensional (2D) carbon material has two $\pi$ electrons and two atoms per unit cell, resulting in a semimetallic electronic band with a conical intersection at the Fermi energy (the $K$ point of the Brillouin zone). Thus charge carriers near the Fermi energy behave like 2D massless relativistic particles exhibiting a linear (photonlike) dispersion relation, which is effectively described by the Dirac equation with Fermi velocity $v_{F} \approx 10^{6} \mathrm{~m} / \mathrm{s}$ as follows: ${ }^{1}$

$$
\left[-i \hbar v_{F} \vec{\sigma} \cdot \vec{\nabla}+U\right] \Psi=i \hbar \frac{\partial \Psi}{\partial t},
$$

where $U$ is the external electric potential and $\vec{\sigma}=\left(\sigma_{x}, \sigma_{y}\right)$ are the Pauli matrices.

This enables an analogy between the quantum wave nature of graphene electronics and the electromagnetic (EM) waves in dielectrics described by Maxwell equations within the electron mean free path scale. For example, graphene electrons can exhibit electronic left-handed materials, ${ }^{2}$ quantum Goos-Hänchen $(\mathrm{GH})$ shift, ${ }^{3,4}$ Bragg reflectors, ${ }^{5}$ and wave guides. ${ }^{6}$ All previous theoretical studies of these properties for graphene were carried out analytically, limiting the analysis to stationary solutions such as finding confined modes ${ }^{6,7}$ or describing plane waves. ${ }^{2-5,8}$ Such descriptions do not provide an understanding of the dynamics of localized electron wave packets, which can be essential in tracing the position of the electron.

This letter addresses the following questions: (1) do the optical-like behavior formulated in the wavelike point of view of the graphene electron remains valid when one includes the particlelike character of spatially localized electron wave packets? (2) Can the graphene electron's exotic tunneling behavior (Klein tunneling) or the GH shift be observed in the time-resolved dynamics? In order to clarify such questions, we developed the "GraFDTD" method to calculate numerically the time evolution of the de Broglie

\footnotetext{
a) Electronic mail: haa@caltech.edu.

${ }^{b)}$ Electronic mail: wag@wag.caltech.edu.
}

wave for the excited graphene electrons. In this letter, we use GraFDTD to investigate the scattering behavior of an electron wave packet at a heterojunction boundary. Then, we compare our results with analytical results based on the plane wave description and with the dynamics of EM waves at the interface of two different dielectric media.

The electronic wave function in graphene is described by a two-component isospinor $\Psi=\left(\psi_{1}, \psi_{2}\right)$ resulting from having two atoms per unit cell. To describe the time evolution of Eq. (1), we discretize the time domain using the velocity Verlet algorithm, which has the virtue that it is a second order symplectic integrator allowing us to sample both $\psi_{1}$ and $\psi_{2}$ simultaneously. The spatial derivatives of $\psi_{1}$ and $\psi_{2}$ are carried out using the finite difference method by discretizing the 2D space with $\Delta x$ and $\Delta y$, yielding a $(M \times N)$ rectangular grid. The detailed time-update equations of $\psi_{1}$ and $\psi_{2}$ are provided as a supplementary material. ${ }^{9}$

This simulation scheme which updates two pseudospin components alternately resembles the finite-difference time-domain (FDTD) simulation method for EM wave modeling ${ }^{10}$ which updates electric field and magnetic field alternately.

Here, we consider a square shaped graphene and choose ordinary Cartesian coordinates with the $x$ and $y$ axes parallel to the sides of the graphene sheet as shown in Fig. 1. Details on the simulation cells are described in the supplementary material. $^{9}$

To achieve a heterojunction, experimentalists have adjusted the gate voltage on materials adjacent to the graphene sheet. In simulations, we set an external electric potential $U$ depending on the incident angle $\theta_{I}$ as $u_{1}$ where $y<x \tan \theta_{I}$ and $u_{2}$ where $y \geq x \tan \theta_{I}$. We use four potential profiles $\left(u_{1}, u_{2}\right)=\left(0,0.5 E_{F}\right),\left(0,1.5 E_{F}\right),\left(0.5 E_{F}, 0\right)$, and $\left(1.5 E_{F}, 0\right)$, denoted as case $1,2,3$, and 4 . The Fermi energy is chosen to be $E_{F}=0.276 \mathrm{eV}$ which leads to $\lambda=h v_{F} / E_{F}=15 \mathrm{~nm}$ for the de Broglie wavelength.

The localized electron (described as a Gaussian wave packet of $50 \mathrm{~nm}$ size) is generated at $y=0$ boundary. ${ }^{9}$ Since 


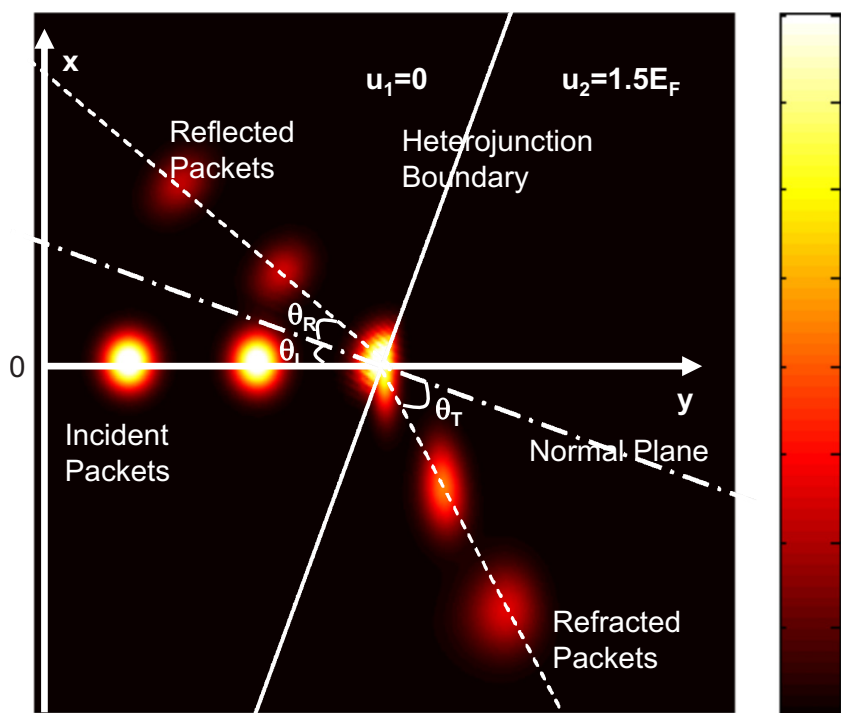

FIG. 1. (Color online) Dynamics of a Gaussian electron wave packet at a heterojunction interface. Snapshots are taken at every $400 \mathrm{fs}$ and displayed simultaneously. Wave packet is colored by the probability density. The incident packet is introduced along the $y$-axis. Physical parameters are chosen to be $\theta_{I}=20^{\circ}$ and $\left(n_{1}, n_{2}\right)=(1,-0.5)$.

the linear dispersion relation is valid within the ballistic transport regime, spatial localization within the mean free path is a more reasonable model of the graphene electron rather than a plane wave description. Experimentally, the graphene system is known to have a mean free path of several $100 \mathrm{~nm},{ }^{11,12}$ much larger than the lattice constant of $0.247 \mathrm{~nm}$. Thus, we consider that a spatial localization of 50 $\mathrm{nm}$ provides a reasonable description of the graphene electrons.

When a de Broglie wave of an electron approaches the heterojunction interface, the electron wave packet is split into two parts, one transmitted and one reflected. By tracking the position of the wave packet, $\langle\mathbf{x}\rangle=\int \Psi^{\dagger} \mathbf{x} \Psi d x d y$, we obtain the angle of reflection $\theta_{R}$ and refraction $\theta_{T}$, as a function of the angle of incidence $\theta_{I}$. The result shows that the law of reflection $\left(\theta_{I}=\theta_{R}\right)$ and the Snell's law $\left(n_{1} \sin \theta_{I}=n_{2} \sin \theta_{T}\right)$ remain valid in the graphene electron system. Here, we introduced the effective refractive index for graphene electrons, $n=1-\left(u / E_{F}\right) .^{2}$

At a heterojunction, we calculate the probability of transmission $T_{\mathrm{GE}}$ by integrating the probability density over the region $y \geq x \tan \theta_{I}$. On the other hand, analytical solutions of $T_{\mathrm{GE}}$ for a electronic plane waves can be expressed in terms of $\theta_{I}$ and $\theta_{T}{ }^{8,13}$

$$
T_{\mathrm{GE}}=\frac{\cos \theta_{I} \cos \theta_{T}}{\cos ^{2}\left[\left(\theta_{I}+\theta_{T}\right) / 2\right]} .
$$

Figure 2 shows how $T_{\mathrm{GE}}$ changes upon varying $\theta_{I}$ from both the simulations (dots) and the theory (solid lines). These results show that the simulations using Gaussian wave packets agree well with plane wave solutions.

Interestingly, we find that $T_{\mathrm{GE}}$ approaches unity at normal incidence regardless of the potential height, which is impossible for the electrons obeying the standard Schrödinger equation. This unique phenomenon is referred as a Klein tunneling. ${ }^{14}$ Indeed, even for the potential wall 1.5 times higher than the electron energy, $T_{\mathrm{GE}}=0.98$ for the normal incidence [see Fig. 2(b)]. Furthermore, we confirmed
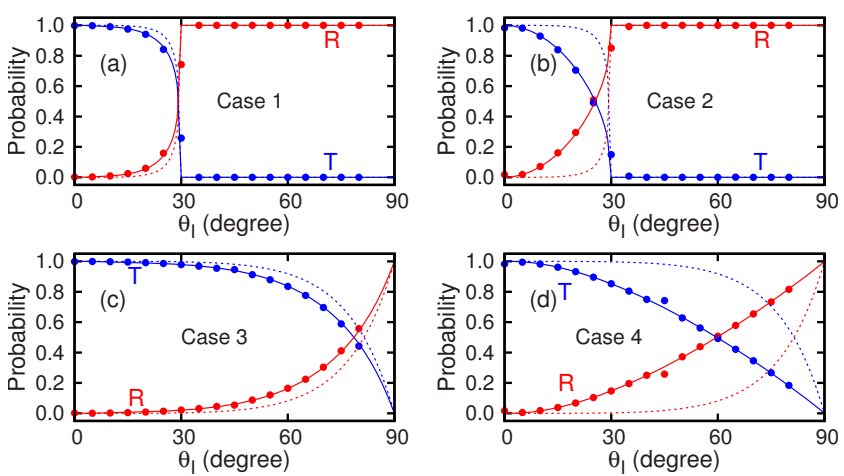

FIG. 2. (Color online) Transmittance (T) and reflectance (R) vs incidence angle $\theta_{I}$ for (a) case 1 , (b) case 2, (c) case 3, and (d) case 4. Simulation results for $T_{\mathrm{GE}}$ and $R_{\mathrm{GE}}$ (circles) agree with the analytic results (solid lines) and are compared with the $T_{\mathrm{EM}}$ and $R_{\mathrm{EM}}$ for EM waves (dotted lines).

that the Klein tunneling happens for the higher potential wall in the limit of infinitesimal grid size. ${ }^{9}$ Recently, conductance experiments have demonstrated Klein tunneling using low energy excited graphene electrons. ${ }^{15,16}$

When the impedances of two optical media are matched, the transmittance becomes identity for normal incidence like the Klein tunneling of graphene electrons. Therefore, this impedance matched interface (IMI) can be regarded as an optical counterpart for the heterojunction of the graphene system. Based on this condition, the transmittances of the TE and TM wave become identical to each other, which is expressed as ${ }^{17}$

$$
T_{\mathrm{EM}}=\frac{\cos \theta_{I} \cos \theta_{T}}{\left[\left(\cos \theta_{I}+\cos \theta_{T}\right) / 2\right]^{2}} .
$$

We notice from Fig. 2 that $T_{\mathrm{GE}}$ and $T_{\mathrm{EM}}$ have a very similar form, having identical critical angles $\theta_{c}=\sin ^{-1}\left(n_{2} / n_{1}\right)$ for total internal reflection and a similar convex decay pattern as $\theta_{I}$ increases. Mathematically, however, $T_{\mathrm{EM}} \geq T_{\mathrm{GE}}$ for $\theta_{I}$ $\in\left[0^{\circ}, 90^{\circ}\right]$ indicating that for the IMI the EM wave transmits better than the graphene electron for the heterojunction boundary. Moreover, $T_{\mathrm{GE}}$ shows a dramatic change depending on the sign of $n$ (i.e., depending on the chirality), while $T_{\mathrm{EM}}$ depends only on the magnitude of $n$.

When the graphene electron undergoes the total internal reflection at the heterojunction boundary, we observe that the center of the Gaussian wave packet is shifted by a distance $d_{\mathrm{GE}}$. This singular behavior is referred as a $\mathrm{GH}$ shift in the graphene system, as proposed recently by Zhao and Yelin ${ }^{3}$ and refined by Beenakker et al. ${ }^{4}$ According to the analytical calculation by Beenakker et al., the $d_{\mathrm{GE}}$ is

$$
d_{\mathrm{GE}}=\frac{\lambda}{2 \pi} \frac{\sin ^{2} \theta_{I}+n}{\sin \theta_{I} \cos \theta_{I} \sqrt{\sin ^{2} \theta_{I}-n^{2}}},
$$

where $n=n_{1} / n_{2}$. We obtain $d_{\mathrm{GE}}$ from our simulations from a linear fit to $\langle\mathbf{x}\rangle$ before and after the reflection at the boundary. Figure 3 shows the dependence of these values on $\theta_{I}$ which agrees well with the Eq. (4) including the sign change in $\mathrm{GH}$ shift around $\theta_{I}=\sin ^{-1} \sqrt{\sin \theta_{c}}=45^{\circ}$ at the p-n boundary. Since this sign change phenomenon was predicted by Beenakker et al. but not from the work of Zhao and Yelin, our simulation supports Beenakker's consideration of the pseudospin degree of freedom. 


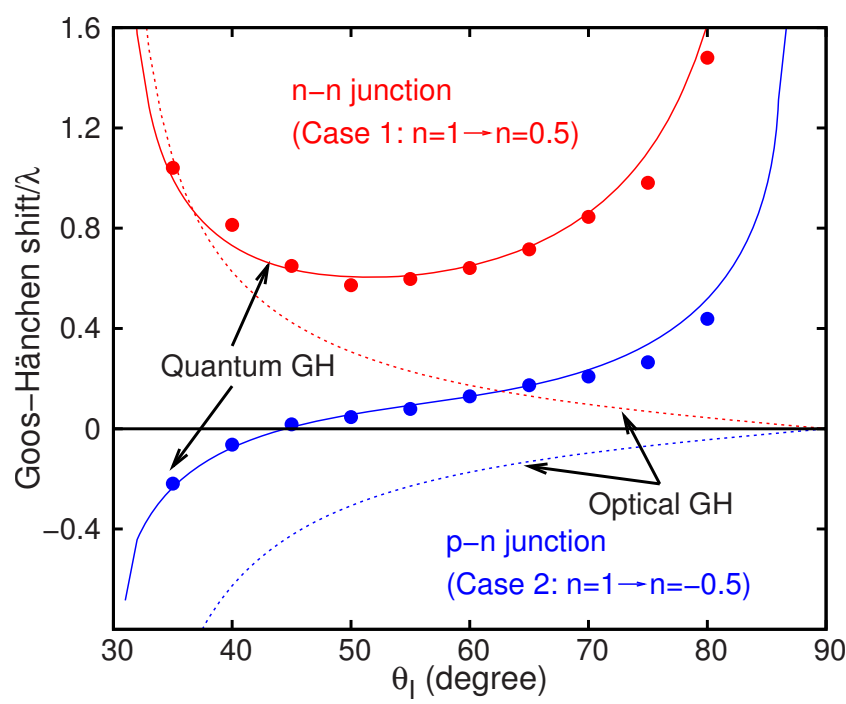

FIG. 3. (Color online) $\mathrm{GH}$ shift vs the incidence angle $\theta_{I}$ for $\left(n_{1}, n_{2}\right)$ $=(1,0.5)$ and $(1,-0.5)$. Simulation results for $d_{\mathrm{GE}}$ (circles) agree with the previous analytical results (solid lines) and are compared with the GH effect for EM waves $d_{\mathrm{EM}}$ (dotted lines).

The Beenakker's analytical calculation considered a Gaussian beam, whose pseudospin components are centered at different positions caused by the transverse momentum localization. In contrast, our simulation considers a more general wave packet, whose pseudospin components are localized at the same position. The excellent correspondence between numerical and analytical results (beyond the slightly different interpretations on the wave packets) supports the presence of quantum $\mathrm{GH}$ effect and the validity of the Eq. (4).

The GH effect, a lateral shift that a linearly polarized light beam experiences in total internal reflection, has been known for optical systems. ${ }^{18}$ It is interpreted in terms of a horizontal energy flow of an evanescent wave in the medium having a lower index of refraction during the total internal reflection. Figure 3 compares $d_{\mathrm{GE}}$ with $d_{\mathrm{EM}}$, the optical $\mathrm{GH}$ shift at IMI, showing that the limiting behavior at $\theta_{I} \rightarrow \theta_{c}$ is analogous for both optical and graphene systems. However, the limiting behavior at $\theta_{I} \rightarrow 90^{\circ}$ is totally different since $d_{\mathrm{EM}}$ is suppressed while $d_{\mathrm{GE}}$ diverges to infinity. In addition, the optical system does not exhibit the sign change behavior. These differences lead to a more restricted incident angle window for the graphene system to exhibit the negative $\mathrm{GH}$ effect compared to the optical systems.

Summarizing, we used the GraFDTD method to study the dynamics of low energy excited graphene electrons at heterojunction boundaries. These numerical simulations lead the demonstrations of Snell's law, negative refraction, Klein tunneling, and quantum GH effect for graphene electrons using temporally and spatially resolved electron wave packets. These results extend the previous plane wave based analytical findings focused on the wavelike character of the graphene electron to the temporal behavior of the electron possessing the particlelike character. Additionally, we thoroughly discuss the similarities and differences of the graphene electron dynamics with their optical counterparts. Each of these is a key issue for graphene based device applications based on the optical analogy framework. ${ }^{2,3}$ As future works, we can now use this validated GraFDTD for investigation of graphene electron dynamics under the nonideal potential shape of gate voltage, scattering behavior at the impurities, and electron transport using waveguides.

This work was supported by DOE LMI EFRC under Grant No. DE-SC0001293 (M.S.J. and H.A.A.) and the Functional Engineered Nano Architectronics (FENA) via the Microelectronics Advanced Research Corporation (MARCO) with the prime award (2009-NT-2048) at UCLA (PI Kang Wang). H.J. and W.A.G. also acknowledge support from the WCU programs through NRF of Korea funded by the MEST (Grant No. R31-2008-000-10055-0). Two of the authors (M.S.J. and H.K.) contributed equally to this work.

${ }^{1}$ A. H. Castro Neto, F. Guinea, N. M. R. Peres, K. S. Novoselov, and A. K. Geim, Rev. Mod. Phys. 81, 109 (2009).

${ }^{2}$ V. V. Cheianov, V. Fal'ko, and B. L. Altshuler, Science 315, 1252 (2007).

${ }^{3}$ L. Zhao and S. Yelin, arXiv:0804.2225v2 (unpublished).

${ }^{4}$ C. W. J. Beenakker, R. A. Sepkhanov, A. R. Akhmerov, and J. Tworzydlo, Phys. Rev. Lett. 102, 146804 (2009).

${ }^{5}$ S. Ghosh and M. Sharma, J. Phys.: Condens. Matter 21, 292204 (2009).

${ }^{6}$ F. M. Zhang, Y. He, and X. Chen, Appl. Phys. Lett. 94, 212105 (2009).

${ }^{7}$ J. M. Pereira, V. Mlinar, F. M. Peeters, and P. Vasilopoulos, Phys. Rev. B 74, 045424 (2006).

${ }^{8}$ M. I. Katsnelson, K. S. Novoselov, and A. K. Geim, Nat. Phys. 2, 620 (2006).

${ }^{9}$ See supplementary material at http://dx.doi.org/10.1063/1.3454909 for detailed time-update equations of $\psi_{1}$ and $\psi_{2}$ and details on the simulation cells.

${ }^{10}$ K. S. Yee, IEEE Trans. Antennas Propag. AP-14, 302 (1966).

${ }^{11}$ X. Du, I. Skachko, A. Barker, and E. Y. Andrei, Nat. Nanotechnol. 3, 491 (2008).

${ }^{12}$ K. I. Bolotin, K. J. Sikes, J. Hone, H. L. Stormer, and P. Kim, Phys. Rev. Lett. 101, 096802 (2008).

${ }^{13}$ V. V. Cheianov and V. I. Fal'ko, Phys. Rev. B 74, 041403 (2006).

${ }^{14}$ O. Klein, Z. Phys. 53, 157 (1929).

${ }^{15}$ A. Young and P. Kim, Nat. Phys. 5, 222 (2009).

${ }^{16}$ N. Stander, B. Huard, and D. Goldhaber-Gordon, Phys. Rev. Lett. 102, 026807 (2009).

${ }^{17}$ M. Born and E. Wolf, Principles of Optics, 7th ed. (Cambridge University Press, 1999), Chap. 1.5 , pp. 38-53.

${ }^{18}$ F. Goos and H. Hänchen, Ann. Phys. 436, 333 (1947). 